\title{
高速铁路腔室耗能型风屏障气动冲击力的 动模型试验研究 ${ }^{*}$
}

\author{
毛 军 ${ }^{1}$ 柳润东 $^{1}$ 郗艳红 $^{1}$ 郭迪龙 $^{2}$ 赵 萌 ${ }^{3}$ \\ (1. 北京交通大学土木建筑工程学院 北京 100044 ; \\ 2. 中国科学院力学研究所 北京 100190 ;
}

3. 内蒙古工业大学能源与动力工程学院 呼和浩特 010051)

\begin{abstract}
摘要: 高速铁路沿线的防风屏障因经常受到列车风和横风的气动冲击作用, 其自身结构稳定性十分重要。利用高速列车动模 型平台进行列车运动对腔室耗能型风屏障产生气动冲击的 $1: 8$ 缩尺模型试验。测试了不同车型的列车以不同车速通过风屏 障区域时, 在风障不同位置处产生的气动压力, 并分析气动压力的变化规律。研究结果表明: 高速列车通过风障区域时, 对 风障各部分均形成了 “正-负-负-正” 的脉动压力; 随着列车车速的增加, 气动冲击压力幅值增大, 头车波和尾车波换向时间 减小, 压力变化率增大; 压力极值及变化率与车速的二次方相关, 不同车速下的压力系数几乎相等; 风障不同高度处压力波 的变化趋势相同, 但幅值不同, 在列车鼻雉高度区域出现最大值; 不同车型的列车在相同车速下对风障气动冲击作用趋势相 同, 但幅值不同, 针体头型列车的气动压力大于子弹头型列车的; 随着风障距轨道中心线距离增大, 列车脉动压力逐渐减小, 其中头车波峰减小最为明显, 与安装间距近似成线性关系。
\end{abstract}

关键词: 高速列车; 动模型试验; 风屏障; 脉动压力

中图分类号: U216

\section{Moving Train Model Experiment Study on the High Speed Railway Chamber Windbreak}

\author{
MAO Jun ${ }^{1}$ LIU Rundong ${ }^{1} \quad$ XI Yanhong ${ }^{1} \quad$ GUO Dilong $^{2} \quad$ ZHAO Meng $^{3}$ \\ (1. The Civil Institute, Beijing Jiaotong University, Beijing 100044; \\ 2. Institute of Mechanics, Chinese Academy of Sciences, Beijing 100190;
}

3. College of Energy and Power Engineering, Inner Mongolia University of Technology, Huhhot 010051)

\begin{abstract}
Installing windbreak along high-speed railway is an effective measure to enhance the safety of high speed train under cross-wind. The experiments were carried out on the high speed train dynamic model platform of the Institute of Mechanics, Chinese Academy of Sciences. The moving high speed train model with a scale ratio of $1: 8$ through the windbreak region with high speed. The different positions' fluctuating pressure of the windbreak caused by different train types and different train speeds were tested. The results show that: when the high-speed train passes through the windbreak region, it forms a positive-negative-negative-positive fluctuating pressure on the windbreak. The trend of the pressure wave at different height of the windbreak is similar, but the amplitude is different. The pressure coefficients at different speeds are almost equal. The maximum pressure value occurs at the train nose height. With the increase of train speed, the amplitude of the fluctuating pressure increases, the reversing time reduces, and the pressure gradient increases. With the different type of the train, the trend of pressure is similar, but the amplitude is different. The aerodynamic pressure of the blunt body head is larger than that of the bullet head. With the distance between the windbreak and the center line increasing, the pressure decreases gradually. The head crest reduces most obviously, and it's linear with distance.
\end{abstract}

Key words: high speed train; moving model; windbreak; fluctuating pressure

\footnotetext{
* 国家重点研发计划课题(2016YFC0802206)、国家自然科学基金 (51278032)和中央高校基本业务费(2017JBM095)资助项目。20180916 收 到初稿, 20190306 收到修改稿
} 


\section{0 前言}

高速列车在大风区运行时, 其横向稳定性明显 恶化, 易发生脱轨、倾覆事故。例如, 我国兰新铁 路自通车至 2010 年因大风引起的列车脱轨、倾覆事 故近 40 起, 货车被吹翻 110 辆, 因大风引起的晚点、 停运造成的损失更是无法计算 ${ }^{[1]}$ 。为保证大风区的 行车安全, 通常在线路一侧或两侧安装挡风屏障, 以减少横风作用在车体上的侧向力和侧翻力矩 ${ }^{[2]}$ 。 同时, 列车周身的气体边界层产生突变, 由此产生 了非定常的压力场和速度场, 这些突变的压力场 和速度场会威胁到风屏障自身的安全稳定性 ${ }^{[3]}$ 。 风屏障除了要承受横风引起的气动荷载之外, 还 会受到列车高速通过时引起的风致气动冲击作 用。列车风在短时间内会产生换向, 在风屏障表面 产生气动冲击力, 引起风障振动和结构内部出现交 变应力, 严重情况下可能导致疲劳破坏问题, 进而 影响列车运行安全。因此, 有必要研究高速列车通 过风屏障区域时产生的气动冲击作用, 以掌握风屏 障所受气动荷载的特性, 为风屏障设计和稳定性评 估提供依据。

列车高速运动时产生了非定常的流场并伴随着 急剧变化的压力场和速度场 ${ }^{[4]}$ 。静模型风洞试验采 用相对运动的原理, 无法模拟出列车自身运动时的 真实流场, 难以准确地还原列车绕流对周围环境的 气动作用和列车交会场景。为此, 近年来, 世界各 地建成了数个高速列车动模型试验平台 (Moving model rig, MMR)。MMR 的优点是: 可模拟列车在 各种线路条件下的绝对运动; 能模拟列车通过风屏 障区、声屏障区、隧道、桥梁、车站等处的情形; 其三, 两列列车会车只能通过 MMR 进行试验 ${ }^{[5]}$ 。 日本东京 Kobayashi 物理研究所建立了全长 $60 \mathrm{~m}$ 的 缩比为 $1 / 30$ 的动模型试验系统, 其中试验段全长 35 $\mathrm{m}$, 它通过高压空气迅速释放到列车模型加速段内 来实现模型加速, 列车模型能够达到的最大速度由 储气罐的体积决定, 设计最高时速为 $500 \mathrm{~km} / \mathrm{h}^{[6]}$ 。 英国 AEA 研究所建立了全长 $146 \mathrm{~m}$ 的缩比为 $1 / 25$ 的动模型试验系统, 其中试验段全长为 $46 \mathrm{~m}$, 它通 过预拉紧的橡胶绳来将列车模型弹射出去, 可进行 模型的明线运行空气动力学研究。试验速度由橡胶 绳和模型质量决定, 最高试验时速为 $277 \mathrm{~km} / \mathrm{h}^{[7]}$ 。 中南大学高铁研究中心建成了全长 $164 \mathrm{~m}$ 的缩比为 $1 / 20 \sim 1 / 16$ 的动模型试验系统, 其中试验段全长为
$50 \mathrm{~m}$, 采用橡皮筋弹射的方式来加速列车模型, 控 制系统可设定模型小车所受拉力的大小，从而得到 所需的试验车速, 最高试验时速为 $500 \mathrm{~km} / \mathrm{h}^{[8]}$ 。

资料表明, 若要得到更准确的列车空气动力学 试验结果, 列车模型缩比应不低于 $1 / 10$, 并达到真 实的运行车速 ${ }^{[9-10]}$, 为此, 本文利用中科院力学所 缩尺比例为 $1: 8$ 的高速列车动模型试验平台, 在试 验平台上安装腔室耗能型风屏障 ${ }^{[11]}$, 开展了高速列 车对风屏障气动冲击作用的试验和分析工作。

\section{1 动模型试验方案及概况}

中国科学院高铁动模型平台利用空气炮弹射装 置，通过调节储气室中的气体压力，可得到不同的 动模型发射速度。为在试验中得到能较好反映风屏 障气动荷载特性的参数, 需要选择合适的传感器、 数采系统等进行试验数据的收集, 从而获得不同工 况下风屏障表面测点处的压力变化。

\section{1 试验平台概况}

高速列车动模型试验平台如图 1 所示, 试验线 全长 $268 \mathrm{~m}$, 其中试验段全长 $60 \mathrm{~m}$, 列车模型缩比 为 $1: 8$, 可将双向对开的高速列车动模型发射至 $400 \mathrm{~km} / \mathrm{h}$ 的速度。

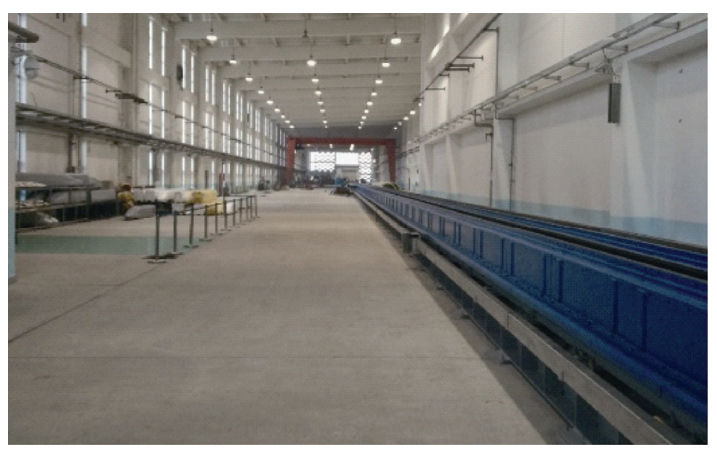

图 1 高速列车动模型试验平台

该动模型试验平台使用空气炮弹射驱动, 当电 控阀门打开时, 压缩空气从储气室中释放, 推动橡 胶活塞加速向前, 活塞通过缆绳与拖车相连接, 拖 车拉动高铁模型达到试验需要的车速, 通过试验段 之后, 列车模型进入减速区, 由于列车本身能量的 消耗以及减速段下方安装的磁减速设施, 可将列车 模型迅速制动 ${ }^{[12]}$ 。

\section{2 试验设备及数据采集系统}

整个动模型试验主要由以下几个部分组成: 控 制终端系统, 空气炮装置, 动模型与轨道系统, 风 障系统, 传感器测试系统与数据采集系统。测试流 程如图 2 所示。 


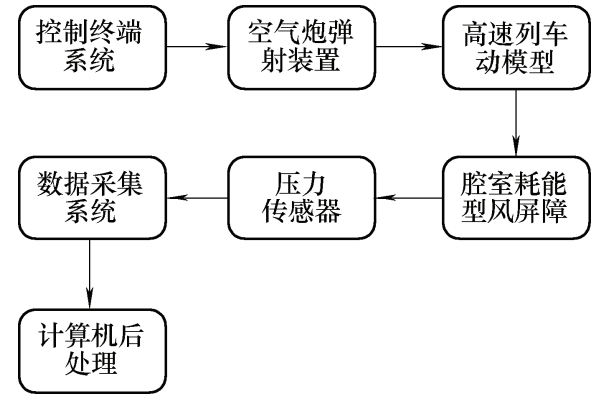

图 2 试验流程图

试验用到的主要仪器设备如下。

(1) 中国科学院高速列车动模型试验平台。

(2) 数采设备: INV3010 型号数据采集器, 24 位高精度数据采集仪, 可进行多通道、高速度、高 精度的振动、噪声、冲击、应变、压力、电压等各 种物理量信号采集。该数采內建信号调理模块, 可 测量多种信号, 便于携带, 适用于试验室、外场、 车载等各种综合性的信号测量。

(3) 传感器: 空气压力传感器, 采用型号为 0815C-15 的 Endvco 空气压力传感器, 该传感器具 有体积小、抗干扰能力强、工作温度范围宽 $(-55$ $\left.125^{\circ} \mathrm{C}\right)$ 、处理电路简单、测量范围广 $(1 \sim 100 \mathrm{~A})$ 、 温度特性好 $\left(-0.1 \% /{ }^{\circ} \mathrm{C}\right)$ 、测量精度高 $(0.1 \% / \mathrm{FS})$ 、灵 敏度极高 $(12 \mathrm{mV} / \mathrm{V} / \mathrm{Oe})$ 等优点。

(4) 腔室耗能型风障系统: 在轨道单侧安装了 腔室耗能型风障系统, 风屏障与列车动模型同样采 用了 $1: 8$ 的缩尺模型, 采用立柱-薄板的约束形式, 风屏障的具体模型如图 5 所示, 风障单板长 500 $\mathrm{mm}$, 高 $375 \mathrm{~mm}$, 腔室厚度为 $20.5 \mathrm{~mm}$, 共 25 个风 障单元，全长 $13.036 \mathrm{~m}$ 。

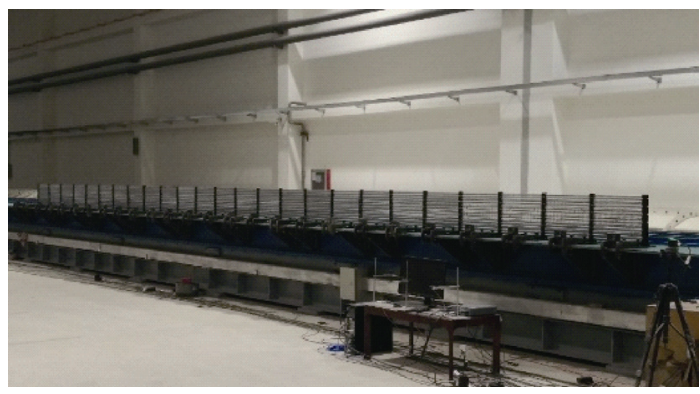

图 3 腔室耗能型风障系统

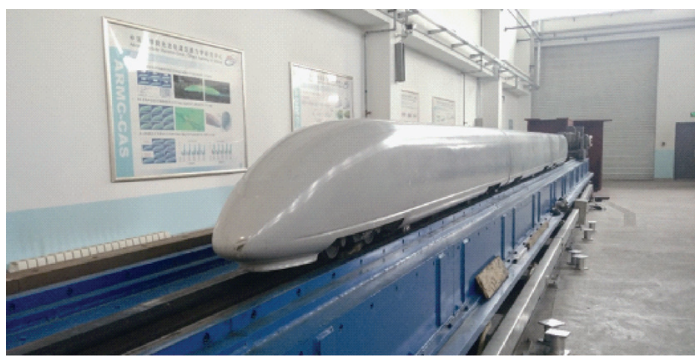

图 4 高速列车动模型
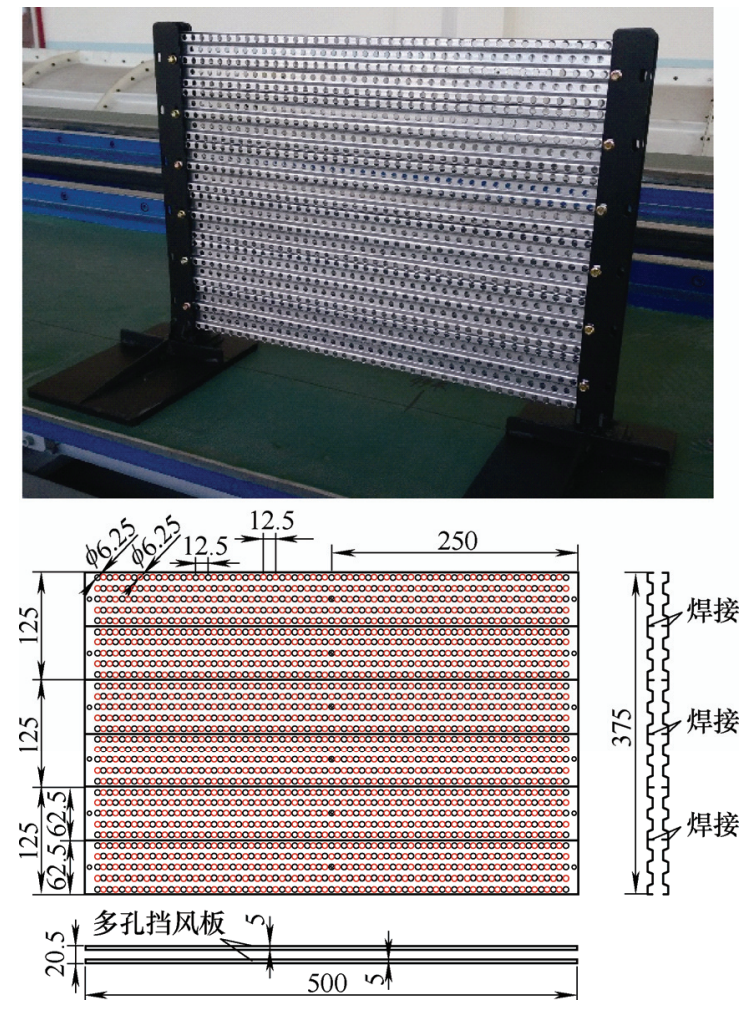

图 5 腔室耗能型风障模型

(5) 高速列车动模型: 使用 $\mathrm{CRH} 380 \mathrm{~A}$ 与 CRH380B 两种高速列车 $1: 8$ 缩尺动模型, 车长为 $10.133 \mathrm{~m}$ 。

\section{3 测量方法的验证}

压力传感器的精度直接关系到试验结果的准确 性, 因此, 在压力传感器安装到风障上之前, 需要 根据现场的试验环境对其进行标定(图 6)。造成误差 主要有两个原因, 一是由于现场环境的温度、湿度 会对传感器的分辨率造成一定的影响; 二是由于数 据采集器的供电电压与传感器的额定电压并不相 同, 因此不能使用传感器出厂自带的分辨率参数。 本试验中采用压力计砝码与静态悬浮球对压力传感 器进行静态标定, 由于风障表面的压力不会超过 \pm 1 $\mathrm{kPa}$, 因此在 $\pm 1 \mathrm{kPa}$ 的范围内逐级进行加载和卸载标 定, 获得校准数据, 以确定压力传感器的灵敏度。

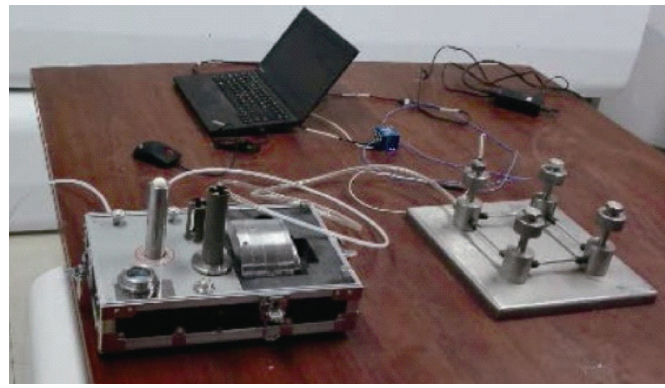

图 6 静态标定试验

\section{4 试验工况}

为了能够了解不同情况下高速列车对风障不同 
位置的气动冲击特性, 试验测试内容主要包含以下 四个方面: 一是测试不同车速下列车对风障的气动 冲击压力; 二是测试列车对风障不同位置处的气动 冲击压力; 三是测试不同车型对风障的气动冲击压 力对比; 四是测试列车对不同远近处的风障的气动 冲击压力。分别发射了 $206.6 \mathrm{~km} / \mathrm{h} 、 220.6 \mathrm{~km} / \mathrm{h} 、 255.6$ $\mathrm{km} / \mathrm{h} 、 272.0 \mathrm{~km} / \mathrm{h} 、 302.0 \mathrm{~km} / \mathrm{h}$ 及 $333.0 \mathrm{~km} / \mathrm{h}$ 六个车 速; 在风障全长共布置 12 个压力监测点, 如图 7 所示, 其中监测点 $P_{4} \sim P_{9}$ 在风障面板高度方向上均
匀分布, 监测点 $P_{1} 、 P_{3} 、 P_{7} 、 P_{11} 、 P_{12}$ 高度相等并 与列车动模型鼻雉等高; $P_{2} 、 P_{6} 、 P_{10}$ 点高度相等; 使用了两种高铁动模型; 改变了五个风障间距; 得 到了不同车速与不同车型下的试验结果。在图 7 中, 列车动模型全长 $10133 \mathrm{~mm}$, 风障全长 $13036 \mathrm{~mm}$, 可保证动模型有全部在风障区间内运行的时段, 轨 道中心距风障内侧表面距离为 $420 \mathrm{~mm}$, 此间距根 据既有风屏障距线路中心线距离经过 $1: 8$ 缩尺后 得到。

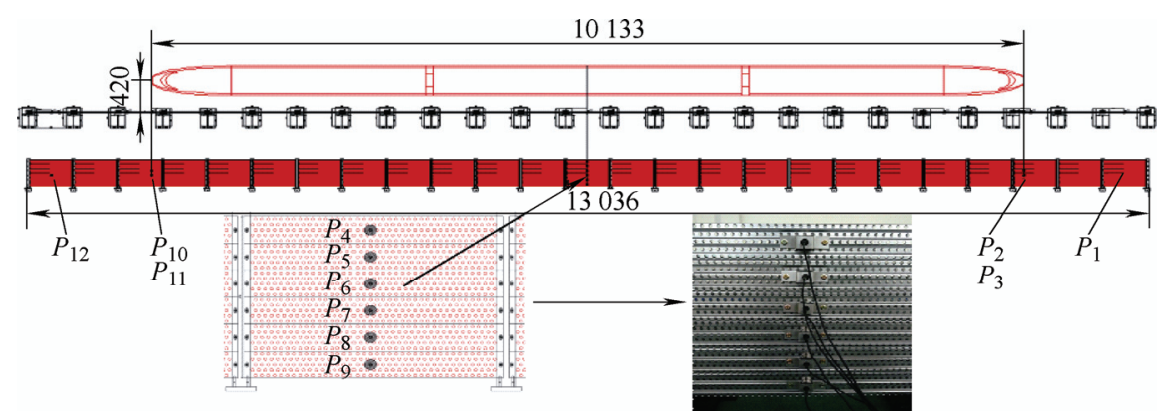

图 7 监测点布置图

\section{2 试验结果及分析}

本次动模型试验主要分析高速列车动车模型通 过风屏障过程中, 对风障的气动冲击情况。

\section{1 不同车速下风障不同位置处的气动压力}

空气作用在风障表面的压强用 $p$ 表示, 其方向 垂直于风障表面, 并以指向风障表面为正方向, 相 对压强 $p_{b}=p-p_{\infty}, p_{\infty}$ 为大气压力, 为便于区分分 析, 定义无量纲参数——障表面压力系数 $C_{p}$, 其 表达式为

$$
C_{p}=\frac{p-p_{\infty}}{0.5 \rho v^{2}}
$$

式中, $v$ 为动模型车速 $(\mathrm{m} / \mathrm{s}), \rho$ 为空气密度。

在与列车鼻雉等高处设置 $P_{1} 、 P_{7}$ 与 $P_{12}$ 监测点, 三个监测点分别位于入口处第一块风障面板, 中间 位置风障面板与出口处最后一块风障面板上, 绘制 列车在不同车速下通过风障区域时三个监测点的压 力系数曲线图, 如图 8 所示。

由图 8 可知, 当动模型高速通过风障区域时, 在风障的入口 $P_{1}$ 、中间 $P_{7}$ 和出口 $P_{12}$ 位置处, 脉动 压力的变化随速度变化的趋势基本相同, 头车携带 先正压后负压, 尾车携带先负压后正压, 共四个压 力波, 在短时间内作用在风障表面, 引起监测点的 压力急剧变化, 其中, 头车作用要明显大于尾车, 且头车所引起的正压在四个压力波中最大。随着速 度的增加, 头车波与尾车波的幅值均逐渐增大, 但

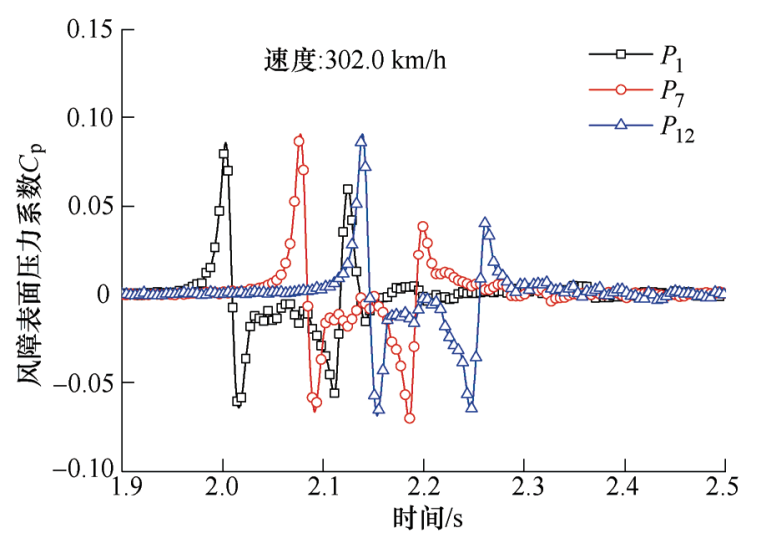

(a)

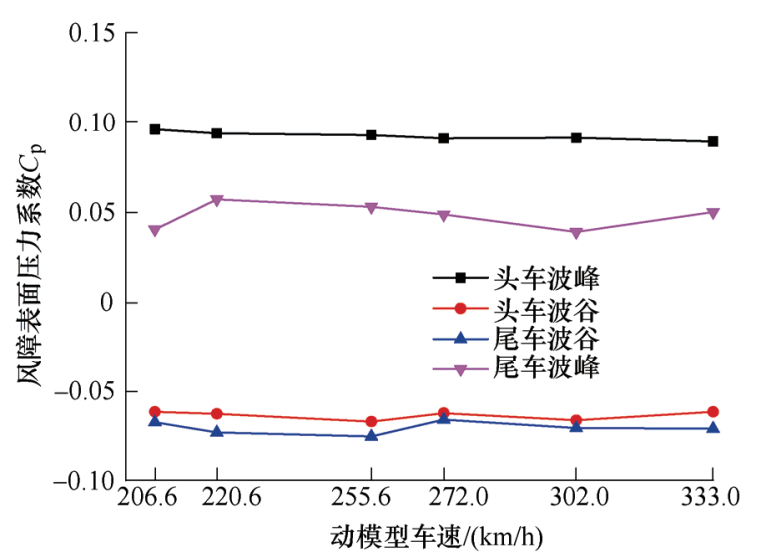

(b)

图 8 不同车速作用下风障表面的脉动压力系数

头车波的变化时间更短, 变化幅值更大, 但经过无 量纲化之后, 六个车速下的压力系数极值基本相等, 只是变化时间会随车速的增加而减小。风障在此过 
程中不仅承受了高强度的列车气动冲击作用, 而且 此压力波短时间内存在两个瞬间换向的过程, 因此 还需要关注此压力波随时间的变化情况, 即压力变
化率, 提取不同车速下的压力极值与压力波变化率, 如表 1 所示, 当高速列车动模型时速为 $333 \mathrm{~km} / \mathrm{h}$ 时, 头车波压力变化率达到 $59.8 \mathrm{kPa} / \mathrm{s}$ 。

表 1 不同车速下的压力极值与压力变化率

\begin{tabular}{|c|c|c|c|c|c|c|}
\hline 车速/(km/h) & 头车波峰/Pa & 头车波谷/Pa & 头车波压力变化率/(Pa/s) & 尾车波谷/Pa & 尾车波峰/Pa & 尾车波压力变化率/(Pa/s) \\
\hline 206.6 & 191.2 & -120.9 & 16426.3 & -133.8 & 78.0 & 10590.0 \\
\hline 220.6 & 215.4 & -138.7 & 20829.4 & -159.9 & 134.9 & 14740.0 \\
\hline 255.6 & 283.5 & -203.3 & 30425.0 & -234.3 & 167.3 & 20080.0 \\
\hline 272.0 & 320.8 & -209.4 & 35346.7 & -224.2 & 164.6 & 25920.0 \\
\hline 302.0 & 389.7 & -271.0 & 47192.9 & -293.7 & 159.5 & 34861.5 \\
\hline 333.0 & 468.0 & -309.2 & 59784.6 & -371.1 & 268.9 & 49230.8 \\
\hline
\end{tabular}

由图 8 和表 1 可得, 列车动模型经过风屏障时, 速度从 $206.6 \mathrm{~km} / \mathrm{h}$ 增加至 $333.0 \mathrm{~km} / \mathrm{h}$, 正压峰值从 191.2 $\mathrm{Pa}$ 增加到 468.0 $\mathrm{Pa}$, 增长率为 $144.8 \%$; 负压 峰值从-133.8 $\mathrm{Pa}$ 增加到-371.1 $\mathrm{Pa}$, 增长率为 $177.4 \%$ 。正压峰值的绝对值大于负压峰值的, 增长 率却小于负压峰值的。头车正压高于负压是由于列 车驶过时对风障表面造成的压缩气流在之后迅速膨 胀时, 会产生一定的能量损失, 因此其形成的负压 回吸作用要小于正压冲击左右; 而尾车正压则由列 车携带的气流冲击导致, 故在四个压力波中绝对值 最小。将车速与压力波峰值及变化率采用最小二乘 法拟合公式可得,

$$
\begin{aligned}
P_{i} & =\alpha_{i}(v / 3.6)^{2} \\
\Delta P_{i-j} & =\beta_{i-j}(v / 3.6)^{2}
\end{aligned}
$$

式中, $P_{1} 、 P_{2} 、 P_{3} 、 P_{4} 、 P_{1-2} 、 P_{3-4}$ 分别代表头车 波峰、头车波谷、尾车波峰、尾车波谷、头车波压 力变化率、尾车波压力变化率, 经计算求得 $\alpha_{1} 、 \alpha_{2}$ 、 $\alpha_{3} 、 \alpha_{4}$ 分别为 $0.0558,-0.0375,-0.04263$, $0.02912 ; \beta_{1-2}$ 与 $\beta_{3-4}$ 分别为 6.4893 与 4.9045 , 式 中的压力变化率通过最大正压减去最大负压再除以 压力变化时间得到, 如式(4)、(5)所示

$$
\begin{gathered}
\Delta P_{1-2}=\frac{P_{1}-P_{2}}{\Delta t_{1}} \\
\Delta P_{3-4}=\frac{P_{4}-P_{3}}{\Delta t_{2}}
\end{gathered}
$$

式中, $\Delta t_{1}$ 为 $P_{1}$ 与 $P_{2}$ 出现的时间间隔; $\Delta t_{2}$ 为 $P_{3}$ 与 $P_{4}$ 出现的时间间隔。

设根据拟合公式得到的压力计算值为 $N_{i}$, 则相 关系数 $R^{2}$ 可由式(6)计算得到

$$
R^{2}=\frac{\sum_{i=1}^{n} P_{i}^{2}-\sum_{i=1}^{n}\left(P_{i}-N_{i}\right)^{2}}{\sum_{i=1}^{n} P_{i}^{2}}
$$

式中， $n=6$, 代表了试验的六个车速。

求解得到的六个相关系数分别为 $R_{1}^{2}=0.9997$, $R_{2}^{2}=0.9983, R_{3}^{2}=0.9977, R_{4}^{2}=0.9794, R_{1-2}^{2}=0.9922$, $R_{3-4}^{2}=0.9755$, 相关系数均在 0.97 以上, 说明此拟 合公式具有较高的可靠性。由此可知脉动压力的峰 值及变化率与速度的平方相关。

\section{2 风障表面不同高度处的气动压力}

在风障中间位置从上到下依次均匀布置六个监 测点, 即图 7 中的 $P_{4} \sim P_{9}$ 点, 监测列车模型高速通 过时的气动冲击作用, 结果如图 9 所示(六个监测点 压力值达到波峰时刻相同，但为在图中区分清楚， 故分别将监测点压力曲线向右进行等差平移)。高速

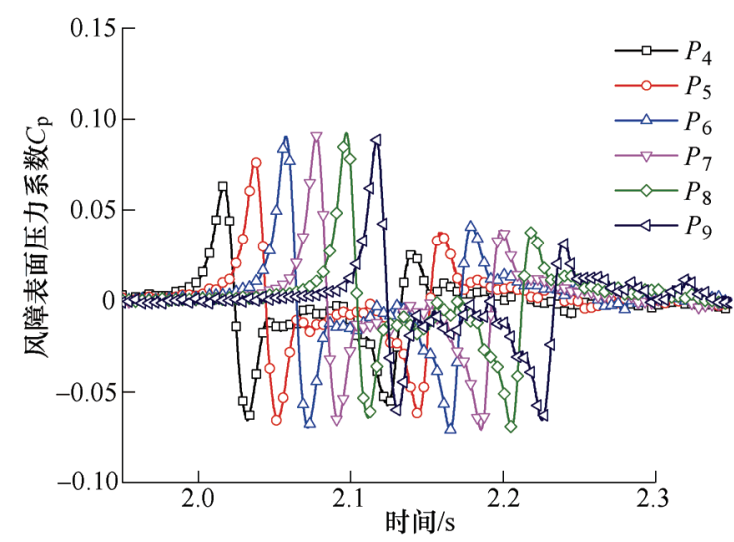

(a) $302.0 \mathrm{~km} / \mathrm{h}$

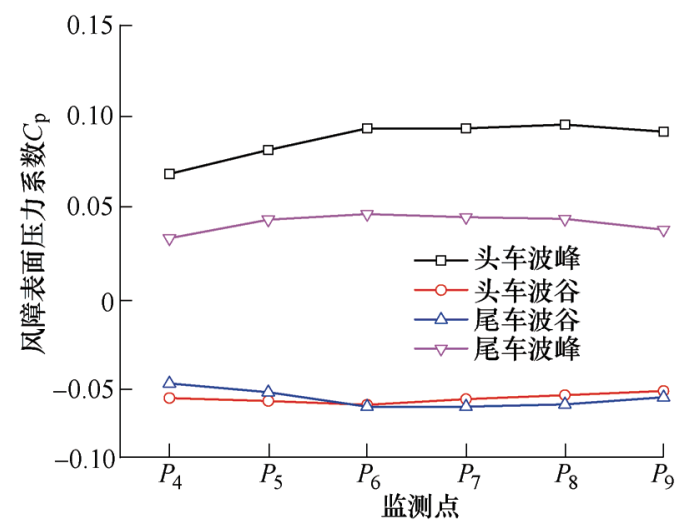

(b)

图 9 不同高度处监测点的脉动压力系数 
列车在风障的不同高度处引起的气动压力在趋势上 保持一致, 但在幅值上随着高度发生变化, 如表 2 所示, 其中, $P_{4} 、 P_{5}$ 点在列车车头上方, 气动压力 最大值出现在 8 号监测点处, 达到 $391 \mathrm{~Pa}$ 。

表 2 风障表面不同高度处气动压力极值 $\mathrm{Pa}$

\begin{tabular}{ccccc}
\hline 监测点 & 头车波峰 & 头车波谷 & 尾车波谷 & 尾车波峰 \\
\hline$P_{4}$ & 274.5 & -272.8 & -239.7 & 117.4 \\
$P_{5}$ & 326.1 & -285.1 & -265.5 & 157.8 \\
$P_{6}$ & 382.8 & -293.3 & -299.5 & 170.1 \\
$P_{7}$ & 382.8 & -283.2 & -298.6 & 162.9 \\
$P_{8}$ & 390.9 & -271.7 & -295.5 & 160.9 \\
$P_{9}$ & 370.4 & -262.4 & -277.9 & 129.8 \\
\hline
\end{tabular}

由表 2 可知, 风屏障表面的脉动压力中间偏下 位置最大, 底部略小, 上部正压比最大处约小 $30 \%$, 这是由于在风障的中部区域, 承受了列车鼻雉处的 驻点高压区对风障产生的气动压力, 而风障底部密 封性较好, 由于地面存在, 较大程度的阻挡了气流 流动, 故中间偏下位置气动压力较大; 而风障上部 区域的开放性较大, 气流流速较大, 故压力要小于 中下部。

\section{3 不同车型作用下风障表面的气动压力}

动模型通过风障区域时所引起的气动压力主要 产生在列车的头车和尾车部位, 而在车体中部则对 监测点形成的是持续较弱的负压, 因此, 此气动压 力与头车和尾车的外形密切相关。如图 10 所示, 本 试验以车头为子弹头形状的 $\mathrm{CRH} 380 \mathrm{~A}$ 为主要试验 车型, 同时也进行了车头更接近钝体的 $\mathrm{CRH} 380 \mathrm{~B}$ 车型的发射, 以比较不同车型所引起的脉动压力的 区别。CRH380B 型车模型的鼻雉高度比 CRH380A 型车低 $25.4 \mathrm{~mm}, \mathrm{~A}$ 型车的鼻雉高度与 $P_{7}$ 点等高, $\mathrm{B}$ 型车的鼻雉高度则介于 $P_{7}$ 点与 $P_{8}$ 点之间, 分别 提取出两个车速下六个高度监测点的压力极值, 并 绘制图 $10 \mathrm{~b}$ 和图 $10 \mathrm{c}$, 图中实线为 $\mathrm{A}$ 型车对监测点 的气动压力系数, 虚线为 $\mathrm{B}$ 型车对监测点气动压力 系数。

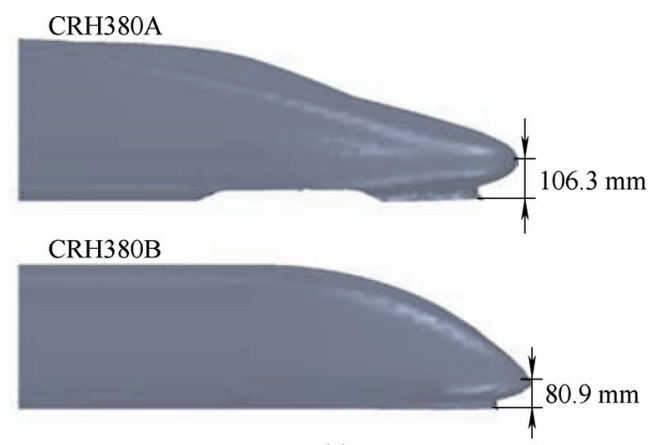

(a)

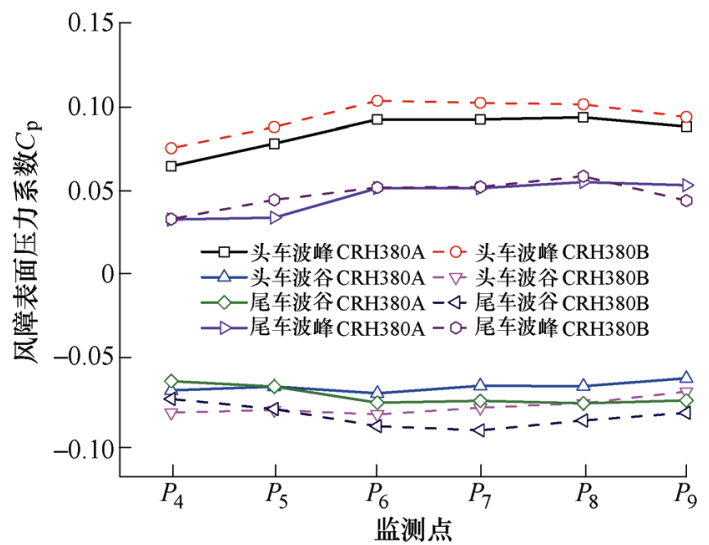

(b) $255 \mathrm{~km} / \mathrm{h}$

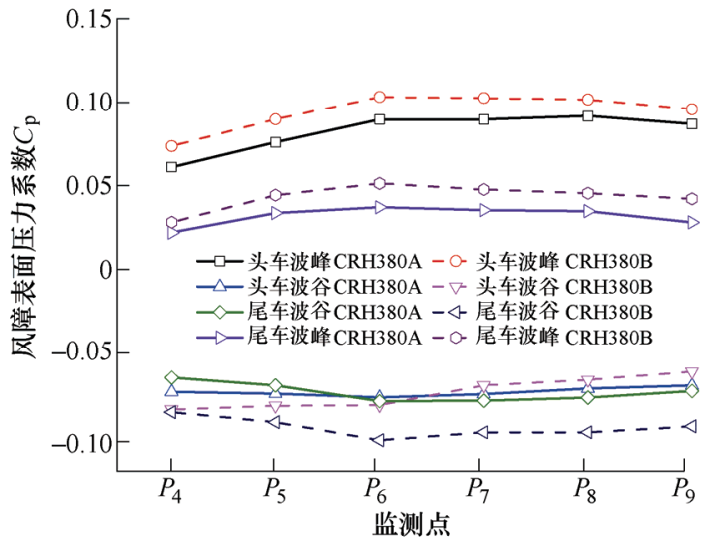

(c) $302 \mathrm{~km} / \mathrm{h}$

图 10 不同车型作用下风障表面的脉动压力系数

两种动模型车型在通过风障区域时, 均对风障 表面产生了 “正-负-负-正” 的脉动压力, 但压力极 值却有不同, 如图 $10 \mathrm{~b}$ 与图 10c 所示, B 型车产生 的脉动压力整体要大于 $\mathrm{A}$ 型车。由于头车波峰压力 系数绝对值最大, 故以 $302 \mathrm{~km} / \mathrm{h}$ 工况的头车波峰为 例, 在 $P_{4} \sim P_{9}$ 六个监测点处, $\mathrm{B}$ 型车分别比 $\mathrm{A}$ 型车 增大约 $19.1 \% 、 17.1 \% 、 14.3 \% 、 13.6 \% 、 10.2 \%$ 及 $9.7 \%$, 原因是由于列车头型的不同, 对周边空气挤压形成 的压力波范围及分布也会不同, $\mathrm{A}$ 型车采用子弹头 型, 在降低列车自身气动阻力的情况下同时也减小 了对风障的气动冲击作用。综上可知, 针体头型的 脉动压力整体要大于子弹头型的脉动压力。

\section{4 不同风障安装间距的气动压力对比}

风障表面所受的气动荷载与风障的安装位置有 很大关系, 风障距轨道距离越远, 则其本身受到的 列车风的冲击荷载就会越小，但是风障安装间距与 列车对风障气动荷载的大小如何变化, 仍需通过具 体数据来分析。通过改变风障的安装位置, 调整其 距轨道中心距分别为 $420 \mathrm{~mm} 、 440 \mathrm{~mm} 、 460 \mathrm{~mm}$ 、 $480 \mathrm{~mm}$ 及 $500 \mathrm{~mm}$, 并分五次发射动模型, 车速为 $302 \mathrm{~km} / \mathrm{h}$, 提取 $P_{7}$ 监测点压力系数随时间变化的数 据, 如图 11 所示(由于监测点到达压力峰值时刻基 
本相同, 为进行区分, 同第 2.2 节一样, 将不同间 距的监测点数据向右进行等差偏移)。

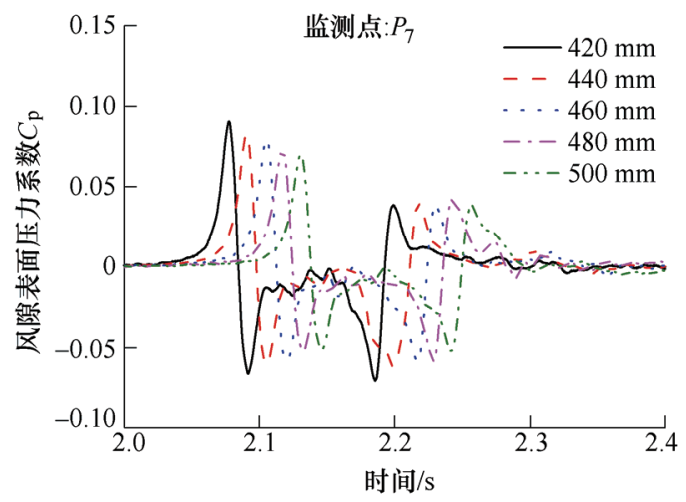

(a)

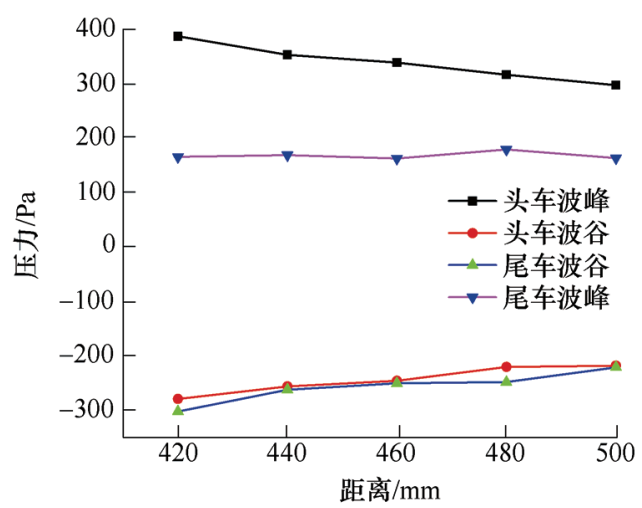

(b)

图 11 不同间距位置处风障表面的脉动压力系数及极值

由图 11 可知, 风障整体外移之后, 随着距轨道 中心线距离的增加, 其气动压力则逐渐减小, 但变 化趋势保持不变。风障高度在列车鼻雉高度及以下 位置所受的气动荷载要高于风障上部位置。如图 $11 \mathrm{~b}$ 所示, 在五个间距下, $P_{7}$ 点头车波正压极值分 别为 $386.3 \mathrm{~Pa} 、 352.4 \mathrm{~Pa} 、 338.3 \mathrm{~Pa} 、 316.1 \mathrm{~Pa}$ 及 297.1 $\mathrm{Pa}$, 在四个压力极值中降低最为明显, 以 $420 \mathrm{~mm}$ 为基准, 随着风障每外移 $20 \mathrm{~mm}$, 则头车波正压极 值分别降低了 $8.8 \% 、 12.4 \% 、 18.2 \% 、 23.1 \%$, 与安 装间距近似成线性关系。

\section{3 结论}

(1) 高速列车经过风障区域时, 在风障各部位 均形成了 “正-负-负-正” 的脉动压力, 其中头车波 为先正压后负压, 尾车波为先负压后正压, 脉动压 力在短时间内发生换向; 随着高速列车车速的增加, 其对风障形成的脉动压力逐渐增大, 同时, 压力波 的换向时间逐渐减小，压力变化率则逐渐增大，脉 动风压力极值与变化率与速度的平方相关, 不同车 速下的压力系数几乎相等。
(2) 高速列车对风障的脉动压力随高度发生变 化, 其中上部开口区域由于流速较大, 故脉动压力 最小，而在列车鼻雉高度附近的压力幅值最大，贴 近地面处略有降低。

(3) 不同车型的高速列车对风障的冲击作用不 同, 子弹头型的车型的冲击作用要小于针体头型; 随着风障距轨道中心距的距离增加, 列车对风障的 气动冲击压力逐渐减小, 头车波峰降低最为明显, 并与风障安装间距近似成线性关系。

由于试验条件限制, 本次试验室内均为无风环 境，而在横风与列车风共同作用下风障的气动荷载 特性, 下一步将通过数值模拟来进行分析。

\section{参 考 文 献}

[1] 刘庆宽, 杜彦良, 乔富贵. 日本列车横风和强风对策研 究[J]. 铁道学报, 2008(1): 82-88.

LIU Qingkuan, DU Yanliang, QIAO Fugui. Train-crosswind and strong wind countermeasure research in Japan[J]. Journal of the China Railway Society, 2008(1): 82-88.

[2] 李人宪, 高速列车气动影响 $[\mathrm{M}]$. 北京: 中国铁道出版 社, 2016.

LI Renxian. Aerodynamic influence of high speed train[M]. Beijing: China Railway Publishing House, 2016.

[3] BAKER C. The flow around high speed trains[J]. Journal of Wind Engineering and Industrial Aerodynamics, 2010, 98(6-7): 277-298.

[4] YANG Guowei. Aerodynamic design for China new high-speed trains[J]. Science China Technological Sciences, 2012, 55(7): 1923-1928.

[5] YANG Qiansuo, SONG Junhao, YANG Guowei. A moving model rig with a scale ratio of $1: 8$ for high speed train aerodynamics[J]. Journal of Wind Engineering and Industrial Aerodynamics, 2016, 152: 50-58.

[6] DOI T, OGAWA T, MASUBUCHI T, et al. Development of an experimental facility for measuring pressure waves generated by high-speed trains[J]. Journal of Wind Engineering \& Industrial Aerodynamics, 2010, 98(1): 55-61.

[7] JOHNSON T, DALLEY S. 1/25 scale moving model tests for the TRANSAERO project[J]. TRANSAERO - A European Initiative on Transient Aerodynamics for Railway System Optimisation, 2002: 123-135.

[8] ZHOU Dan, TIAN Hongqi, ZHANG Jian, et al. Pressure transients induced by a high-speed train passing through a station[J]. Journal of Wind Engineering \& Industrial Aerodynamics, 2014, 135: 1-9.

[9] SCHETZ J A. Aerodynamics of high speed trains[J]. Annu. Rev. Fluid Mech., 2001(33): 371-414. 
[10] 田红旗, 列车空气动力学 $[\mathrm{M}]$. 北京: 中国铁道出版社, 2007.

TIAN Hongqi. Train aerodynamics[M]. Beijing: China Railway Publishing House, 2007.

[11] 毛军, 郗艳红, 高亮, 等. 腔室耗能型高速铁路风障的 减载抗风性能 $[\mathrm{J}]$. 机械工程学报, 2014(4): 99-106.

MAO Jun, XI Yanhong, GAO Liang, et al.Wind resistance performance of the windbreak with air chambers dissipating energy applied in high-speed railway[J].
Journal of Mechanical Engineering, 2014, 4(4): 99-106.

[12] YANG, Qiansuo, SONG Junhao, LI Duo, et al. Train model acceleration and deceleration[J]. Science China Technological Sciences, 2013, 56(3): 642-647.

作者简介: 毛军, 男, 1966 年出生, 博士, 教授, 博士研究生导师。主 要从事高速列车空气动力学和火灾科学研究。

E-mail: jmao@bjtu.edu.cn

柳润东(通信作者), 男, 1990 年出生, 博士。主要从事高速列车空气动 力学研究。

E-mail: 14115264@bjtu.edu.cn 